\title{
EFEITOS DA SUPLEMENTAÇÃO DE RESVERATROL NA SAÚDE HUMANA: UMA REVISÃO INTEGRATIVA
}

\section{EFFECTS OF RESVERATROL SUPPLEMENTATION ON HUMAN HEALTH: AN INTEGRATIVE REVIEW}

\author{
Luane Aparecida AMARAL ${ }^{1}$, Ingrid Gabriela de Oliveira TONINI' ${ }^{2}$, Isabela Cristina \\ MEDINO², Brenda Alana RIBAS $^{2}$, Thiécla Katiane Rosales SILVA ${ }^{3}$, Catiuscie Cabreira \\ da Silva TORTORELLA ${ }^{3}$
}

1 - Nutricionista. Mestranda do Programa de Pós-Graduação em Saúde e Desenvolvimento na Região Centro Oeste da Universidade Federal de Mato Grosso do Sul

2 - Nutricionista. Universidade Estadual do Centro Oeste - UNICENTRO

3 - Docente. Universidade Estadual do Centro Oeste - UNICENTRO

\section{RESUMO:}

O resveratrol é uma fitoquímico de origem vegetal, pertencente à família Stilbenoid estilbenoide e encontra-se nas uvas, amendoins, plantas medicinais e vinho. Este componente tem sido amplamente explorado por sua ação anticancerígena, anti-inflamatória, cardioprotetora, neuroprotetora e efeitos antidiabéticos. Assim, o objetivo deste estudo foi discutir os achados de publicações que abordam os benefícios da suplementação de resveratrol na saúde. Realizou-se uma revisão integrativa em seis etapas: seleção da questão norteadora; definição das características das pesquisas primárias; seleção das pesquisas que compuseram a amostra; análise dos artigos; interpretação dos resultados e exame crítico dos achados. Foram identificados 100 artigos, dos quais cinco foram selecionados. Quatro artigos foram publicados em língua inglesa e um em periódico nacional. A maior parte dos artigos encontrados comprovaram que o resveratrol possui papel benéfico na redução dos riscos de desenvolver doenças e na manutenção da saúde, confirmando sua função como fator protetor contra inúmeras doenças, como obesidade, diabetes e câncer, e, portanto, a suplementação deste polifenol auxilia na melhora da qualidade de vida dos indivíduos.

Palavras-chave: polifenóis, substâncias protetoras, antioxidantes

\section{ABSTRACT:}

Resveratrol is a plant-derived phytochemical belonging to the Stilbenoid estilbenoide family and found in grapes, peanuts, medicinal plants and wine. This component has been extensively explored for its anticancer, anti-inflammatory, cardioprotective, neuroprotective and anti-diabetic effects. Thus, the aim of this study was to discuss the findings of publications that address the health benefits of resveratrol. An integrative review was carried out in six stages: selection of the guiding question; Definition of characteristics of primary research; Selection of the surveys that composed the sample; Analysis of articles; Interpretation of results and critical examination of the findings. We identified 100 articles, of which five were selected. Four articles were published in English and one was published in the national journal. Most articles found that resveratrol has a beneficial role in reducing the risk of developing diseases and maintaining health, confirming its role as a protective factor against numerous diseases, such as obesity, diabetes and cancer, and therefore, the supplementation of this polyphenol helps to improve quality of life of individuals.

Keys words: polyphenols, protective agents, antioxidants 


\section{INTRODUÇÃO}

O consumo adequado de frutas e hortaliças têm sido apontando como um fator protetor contra o risco do desenvolvimento de doenças crônicas não transmissíveis, por outro lado, o alto consumo de alimentos processados associa-se ao risco de doenças relacionadas a má nutrição (ANTUNES; ARAÚJO, 2000; CHANG; SHEEN; LEI, 2015; BRASIL, 2016). Neste contexto, uma dieta rica e amplamente variada em frutas e vegetais tem papel importante em uma dieta saudável, devido ao teor de fitoquímicos presentes (CHANG; SHEEN; LEI, 2015).

Dentre os fitoquímicos mais estudados, destaca-se o resveratrol $(3,5,4$ ' trihydroxystilbene). O resveratrol é uma fitoalexina polifenol de origem vegetal, pertencente à família Stilbenoid estilbenoide e encontrado nas uvas, amendoins, plantas medicinais e processados como o vinho. Vários fungos, incluindo Botryosphaeria, Penicillium, Cephalosporium, Aspergillus, Geotrichum, Mucor e Alternaria, também são importantes fontes de resveratrol. As concentrações destes fenólicos podem variar, dependendo da espécie da planta (AGGARWAL et al., 2004; SHI et al., 2012; THUC et al., 2012).

Devido a variação de concentrações de resveratrol em plantas é difícil prever a quantidade exata do mesmo, pois sua biossíntese pode ser afetada por vários fatores. Levando em consideração esse fato, a maioria dos estudos tem trabalhado com suplementação de resveratrol por meio de cápsulas e tabletes, pois, muitas vezes não é possível ofertar a quantidade ideal pela dieta, surgindo a necessidade de suplementação (MATOS et al., 2012; CHANG et al., 2015; LEAL et al., 2017).

O resveratrol tem sido amplamente explorado por sua ação anticancerígena, antiinflamatória, cardioprotetora, neuroprotetora e efeitos antidiabéticos indicada por diversos estudos (KIM et al., 2007; THUC et al., 2012). O potencial anti-cancerígeno do resveratrol advém de sua capacidade de interromper a proliferação celular de células tumorais, restaurar a apoptose e regular a expressão de genes específicos da carcinogênese. Além disso, também impede a ativação de pró-carcinogênicos, inibindo uma série de eventos obrigatórios para o desenvolvimento tumoral (HSIEH e WU, 2010). Assim, este estudo teve por objetivo realizar uma revisão de literatura sobre os benefícios da suplementação de resveratrol na saúde.

\section{METODOLOGIA}

Trata-se de um estudo de revisão integrativa, o qual foi dividido em seis etapas: 
seleção da questão norteadora; definição das características das pesquisas primárias; seleção das pesquisas que compuseram a amostra; análise dos artigos; interpretação dos resultados; e o exame crítico dos achados, com a seguinte questão norteadora: "Quais os registros em publicações científicas sobre os efeitos da suplementação de resveratrol?"

A identificação do objeto de estudo foi realizada incluindo todos os artigos sobre o resveratrol publicados no período de $1^{\circ}$ de janeiro de 2012 a 20 de novembro de 2016 e indexados no National Library of Medicine (Medline), Literatura Latino-Americana de Ciências da Saúde (Lilacs) e Scientific Electronic Library On Line (Scielo), visando atender a recomendação da literatura de que se busquem diferentes fontes para o levantamento de publicações.

Os descritores em português e inglês utilizados para seleção de artigos foram: resveratrol, resveratrol e doenças crônicas não transmissíveis/resveratrol and Chronic noncommunicable diseases. Os critérios de inclusão foram: artigos experimentais (in vitro e in vivo); em português e inglês com os resumos disponíveis nas bases de dados supracitadas no período estabelecido; e ainda que pudessem responder à questão norteadora. A análise de dados foi realizada de forma qualitativa.

\section{RESULTADOS E DISCUSSÃO}

Foram identificados 100 artigos a partir dos termos mesh/desc, dos quais foram selecionados cinco de acordo com os demais critérios de seleção. Quatro artigos foram publicados em língua inglesa e um foi publicado em periódico nacional (Tabela 1).

Com relação à metodologia, os artigos possuem abordagem quantitativa e qualitativa do tipo descritiva. Os estudos selecionados utilizaram as análises bioquímicas, histológicas e antropométricas para reforçarem seus resultados.

O estudo de Matos et al., (2012) objetivou discutir os efeitos do resveratrol como fator anti-inflamatório e antiaterogênico. O estudo foi realizado com coelhos, divididos em dois grupos: um grupo controle e um grupo resveratrol, sendo que todos os animais receberam dieta hipercolesterolêmica. Neste estudo, esse polifenol apresentou efeito antiinflamatório e antiaterogênico significativo.

Turkez e Aydin (2013) avaliaram os efeitos genéticos do resveratrol em diferentes concentrações, bem como sua ação na genotoxicidade induzida por permetrina. Como resultado, eles encontraram que a associação entre permetrina e resveratrol, quando comparada com o uso destas substâncias isoladas, reduziram significativamente as 
formações de troca de cromátides irmãs, concluindo, assim, que o resveratrol pode ser usado como suplemento dietético, fazendo parte dos alimentos funcionais na prevenção de muitas doenças, incluindo tumores.

Outro estudo in vitro realizado por Noriega-González et al., (2015) investigou a suplementação de resveratrol na obesidade, por meio da avaliação desse composto na atividade mitocondrial e morfologia de adipócitos maduros diferenciados. Neste estudo, o resveratrol, em doses de 25 e $50 \square \mathrm{M}$ durante 48 horas, promoveu a diminuição do conteúdo lipídico, provavelmente devido a um aumento na atividade mitocondrial causado pela exposição a esse polifenol, sendo que houve um declínio da atividade dessa organela ao final das 48 horas.

Luciano et al., (2014) avaliaram o efeito do resveratrol sobre a via de sinalização da insulina e melhora do quadro inflamatório no miocárdio de ratos Wistar obesos induzidos pela dieta. A partir dos resultados, observou-se que a intervenção com o resveratrol reduziu a resistência à insulina nos animais. Além disso, houve redução dos níveis proteicos do fator de necrose tumoral alfa e de moléculas envolvidas com a transdução do sinal próinflamatório. Portanto, a suplementação de resveratrol tem papel na resposta a insulina e na proteção do miocárdio.

Barbosa, Pimenta e Almeida (2016) avaliaram in vivo o efeito radioprotetor do resveratrol sobre os dentes incisivos de ratos irradiados. A pesquisa foi realizada com ratos Wistar adultos, divididos em quatro grupos: controle (não recebeu medicação nem radiação), controle irradiado (recebeu uma dose única de irradiação), resveratrol (recebeu solução de resveratrol) e resveratrol irradiado (recebeu solução de resveratrol e irradiação). Foi observado efeito deletério da radiação proveniente de raio- $X$ na formação dos dentes, todavia o resveratrol não demonstrou efeito radioprotetor para os dentes no estudo em questão.

Além do resveratrol, outros fitoquímicos tem potencial sobre diversas doenças, um deles é a quercetina que tem demonstrado papel terapêutico na prevenção e tratamento de diferentes doenças crônicas, incluindo doenças cardiovasculares e neurodegenerativas, bem como câncer (RUSSO et al., 2012, SERBAN et al., 2016).

O ácido gálico também é um composto fenólico como o resveratrol e a quercetina e apresentou ação anti-metastática e anti-inflamatória em modelos experimentais (HO et al., 2010; HSIANG et al., 2013).

Vale ressaltar que esses fitoquímicos vem sendo testados em células e animais e não se pode concluir que esses mesmos resultados serão vistos em humanos, gerando a necessidade de mais estudos que assegurem seu uso e assim aplicação em humanos. 


\section{CONCLUSÃO}

A partir da análise de estudos que abordam os efeitos da suplementação de resveratrol na saúde humana e na diminuição dos riscos do desenvolvimento de doenças, pode-se inferir que esse composto fenólico apresenta uma considerável contribuição à saúde humana devido a sua ação antioxidante, anti-inflamatória e anticancerígena reportada nos artigos encontrados. Sugere-se que mais estudos sejam desenvolvidos usando os modelos, celular e animal, antes da aplicação em humanos, para elucidar os benefícios e dosagem na suplementação do resveratrol como formulação fitoquímica no organismo humano.

\section{REFERÊNCIAS}

AGGARWAL, B. B.; BHARDWAJ, A.; AGGARWAL, R. S.; SEERAM, N. P.; SHISHODIA, S.; TAKADA Y. Role of resveratrol in prevention and therapy of cancer: preclinical and clinical studies. Anticancer Research, v. 24, n. 5, p. 2783-840, 2004.

ANTUNES, L. M. G.; ARAÚJO, M. C. P. Mutagenicidade e antimutagenicidade dos principais corantes para alimentos. Revista de Nutrição, v. 13, n. 2, p. 81-8, 2000.

BARBOSA, G. L. R.; PIMENTA, L. A.; ALMEIDA, S. M. Micro-CT evaluation of the radioprotective effect of resveratrol on the mandibular incisors of irradiated rats. Braz Oral Res [online], v. 30, n. 1, 2016.

CHANG, H. P.; SHEEN, L. Y.; LEI Y. P. The protective role of carotenoids and polyphenols in patients with head and neck cancer. Journal of the Chinese Medical Association, v. 78, n. 1, p. 89-95, 2015.

HSIEH, T.C.; WU, J. M. Resveratrol: Biological and pharmaceutical properties as anticancer molecule. Biofactors, v. 36, n. 5, p.360-9, 2010.

BRASIL, Instituto Nacional de Câncer José Alencar Gomes da Silva (INCA). Estimativa 2016: incidência de câncer no Brasil. Disponível em: http://www.inca.gov.br/estimativa/2016/. Acesso em: 14 de dezembro de 2016. 
KIM, Y. A.; KIM, G. Y.; PARK, K. Y.; CHOI, Y. H. Resveratrol inhibits nitric oxide and prostaglandin E2 production by lipopolysaccharide-activated C6 microglia. Journal of Medicinal Food, v. 10, p. 218-224.

LUCIANO, T. F.; MARQUES, S. O. PIERI, B. L. S.; SOUZA, D. R.; LIRA, F. S.; SOUZA, C. T. Resveratrol reduces chronic inflammation and improves insulin action in the myocardium of high-fat diet-induced obese rats. Revista de Nutrição, v. 27, n. 2, p. 151-159, 2014.

MATOS, R. S.; BARONCINI, L. A. V.; PRÉCOMA, L. B.; WINTER, G.; CARON, P. H. L.; KAIBER, F.; PRÉCOMA, D. B. Resveratrol Provoca Efeitos Antiaterogênicos em um Modelo Animal de Aterosclerose. Resultados da pesquisa. Arquivos brasileiros de cardiologia, v. 98 , n. 2, p. 136-142, 2012.

NORIEGA-GONZÁLEZ, J. E.; CHIRINO, Y. I.; MATA-MIRANDA, M. M.; VÁZQUEZZAPIÉN, G. J.; SÁNCHEZ-MONROY, V. Effect of Resveratrol on Mitochondrial Activity in Differentiated Mature Adipocytes. International Journal of Morphology., v. 33, n. 3, p. 1085-1092, 2015.

SHI, J. L.; ZENG, Q.; LIU, Y. L.; PAN, Z. L. Alternaria sp. MG1, a resveratrol-producing fungus: isolation, identification, and optimal cultivation conditions for resveratrol production. Applied Microbiology and Biotechnology, v. 95, p. 369-379, 2012.

THUC, L. C.; TESHIMA, Y.; TAKAHASHI, N.; SAIKAWA, T. Inhibition of $\mathrm{Na+-H}+$ exchange as a mechanism of rapid cardioprotection by resveratrol. British Journal of Pharmacology, v. 166, n. 6, p. $1745-1755,2012$.

TURKEZ, H.; AYDIN, E. The Genoprotective Activity of Resveratrol on PermethrinInduced Genotoxic Damage in Cultured Human Lymphocytes. Brazilian Archives of Biology and Technology., v. 56, n. 3, p. 405-411, May/June 2013. 